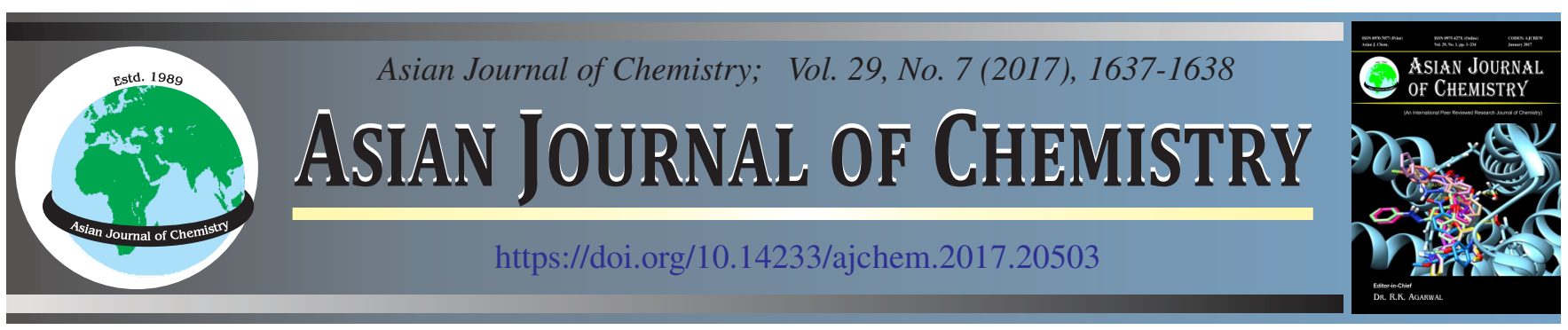

NOTE

\title{
Identification of Phytocomponents in Methanolic Extract of Cuscuta reflexa Grown on Acacia nilotica Host Plant Through GC-MS Analysis
}

\author{
Pooja Saini ${ }^{1}$, Ekta Menghani ${ }^{1, *}$ and Rekha Mithal ${ }^{2}$
}

${ }^{1}$ Department of Biotechnology, JECRC University, Jaipur-302022, India

${ }^{2}$ Department of Chemistry, JECRC University, Jaipur-302022, India

*Corresponding author: E-mail: ekta.menghani@jecrcu.edu.in

\begin{abstract}
In present work, attempts were made to identify different phytochemicals present in the methanol extract of Cuscuta reflexa grown on Acacia nilotica host plant. GC-MS analysis of crude methanol extract was performed to identify different phytochemicals present in the Cuscuta reflexa. In present methanolic extract of Cuscuta reflexa pentanoic acid, 5-hydroxy-, 2,4-di-t-butylphenyl esters, 1-hexanol, 5methyl-2-(1-methylethyl), phthalic acid, isobutyl undecyl ester, octadecanoic acid and celidoniol are identified with major peaks in the GC-MS analysis. Most of these compounds have potentiality to cure various diseases. Cuscuta reflexa plant can be used for the isolation and characterization of new chemical compounds that can be used as future drugs against various life threatening diseases.
\end{abstract}

Keywords: Cuscuta reflexa, GC-MS Analysis, Phytochemicals, Medicinal plant.

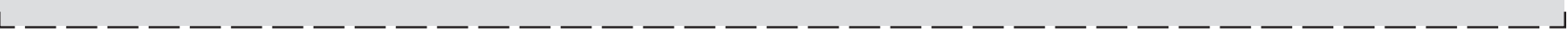

Cuscuta reflexa is a parasitic plant, which grows as weed plant and become a threat for the farmers by its parasitism nature over the crop plant that destroys crop fields [1]. Although this plant becomes a trouble for the crop cultivars but still Cuscuta reflexa have many medicinal activities and called as miracle medicinal plant in the ethno botany.

Cuscuta reflexa possess antibacterial, antiviral, anti-inflammatory, antidiabetic, antifungal, antitumor, hepatoprotective, antifungal activities [2]. This parasitic plant has alkaloids, steroids and flavonoids giving it strong antioxidant potential. Cuscuta reflexa grows over the host plant in parasitic manner and sucks nutrients from the host so it also showed some activities depends on the nature of its host plant.

In the present, Cuscuta reflexa has been studied for the presence of different bioactive compounds. Cuscuta reflexa grown on Acacia nilotica host plant were studied by Gas chromatography mass spectroscopy. GC-MS is an advance technique for the identification of different chemical compounds present in the crude mixture of compounds [3].

Sample plant Cuscuta reflexa grown on Acacia nilotica host plant was collected from the local area of Jhotwara area, Jaipur, Rajasthan. This sample were authenticated and was given identification number and submitted in Ethno-medicinal Herbarium, Centre with potentials of Excellence funded by DST, JECRC University, Jaipur, India. Further, voucher specimens of Cuscuta reflexa was deposited at herbarium of University of Rajasthan, Jaipur, India and was verified by senior taxonomist of department and provided with accession no. RUBL211577.

Processing of plant material: Plant material was collected from the field and screen for the presence of any foreign material. Collected plant sample was shed dried and then grinded. Sample was grinded to powder form and then extraction procedure was followed.

Extraction of plant sample: Methanol extract of dried plant material was prepared by in vitro hot extraction technique with powdered plant material $(100 \mathrm{~g})$. Crude plant extracts was prepared using methanol as a solvent at $10-20{ }^{\circ} \mathrm{C}$ with continue stirring for maximum yield. Then homogenate was filtered and cooled and then concentrated by vacuum distillation method using rotavap. The concentrated plant extract was further used for experimental procedures [4,5].

GC-MS analysis of plant extract: The plant extract was subjected to GC MS analysis to identify the various bioactive compounds present. The sample was analyzed in GCMSQP2010 Plus from Jawahar Lal Nehru University (JNU) India.. An aliquot of $2 \mu \mathrm{L}$ of methanol extract was injected into the column, injector temp. $260{ }^{\circ} \mathrm{C}$ with a split ratio of 10:0, Oven temperature program initial temperature is $100^{\circ} \mathrm{C} .250{ }^{\circ} \mathrm{C}$ for 5 min, ramp $30 \mathrm{~min}$ to $280^{\circ} \mathrm{C}$, hold $69.98 \mathrm{~min}$, ACQ Mode 
TABLE-1

LIST OF COMPOUNDS IDENTIFIED FROM GCMS ANALYSIS OF Cuscuta reflexa METHANOL EXTRACT

\begin{tabular}{cccc}
\hline Peak & Retention time $(\mathrm{min})$ & Compound name & m.f. \\
\hline 1 & 5.785 & Undecane & $\mathrm{C}_{13} \mathrm{H}_{28}$ \\
2 & 8.545 & Pentadecane & \\
3 & 10.133 & Pentanoic acid, 5-hydroxy-, 2,4-di-t-butylphenyl esters & $\mathrm{C}_{14} \mathrm{H}_{30} \mathrm{O}_{3} \mathrm{~S}$ \\
4 & 13.034 & 1-Hexanol, 5-methyl-2-(1-methylethyl) & $\mathrm{C}_{9} \mathrm{H}_{28} \mathrm{O}_{4}$ \\
5 & 14.875 & Phthalic acid, isobutyl undecyl ester & $\mathrm{C}_{10} \mathrm{H}_{22} \mathrm{O}$ \\
6 & 17.027 & Octadecanoic acid & $\mathrm{C}_{23} \mathrm{H}_{36} \mathrm{O}_{4}$ \\
7 & 21.500 & Celidoniol & $\mathrm{C}_{21} \mathrm{H}_{44} \mathrm{O}_{3} \mathrm{~S}$ \\
\hline
\end{tabular}

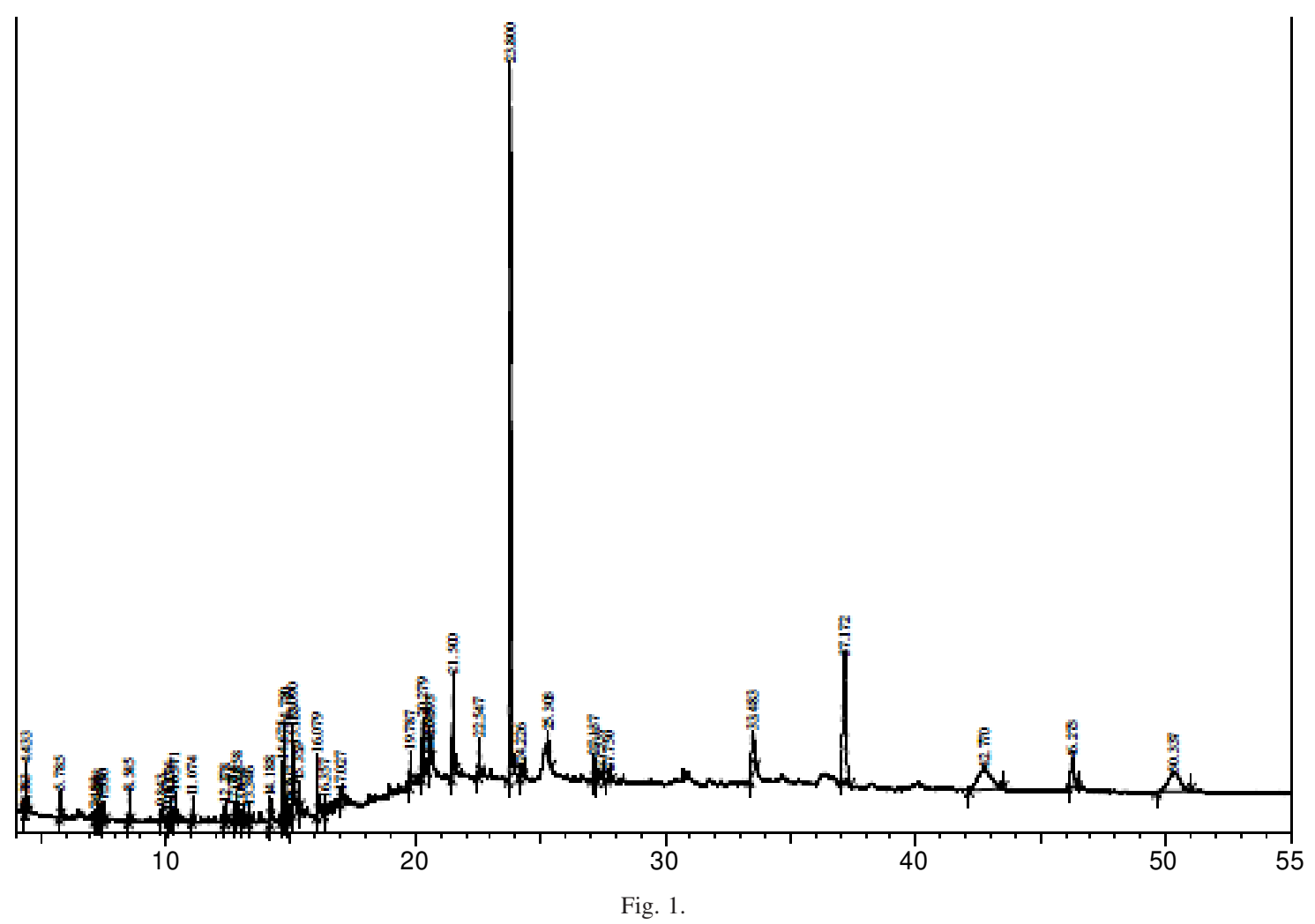

Scan: 40 to $600 \mathrm{~m} / \mathrm{z}$, Column flow is $1.21 \mathrm{~mL} / \mathrm{min}$ and total flow is $16.3 \mathrm{~mL} / \mathrm{min}$. Flow control with linear velocity which $40.9 \mathrm{~cm} / \mathrm{s}$.

Identification of bioactive compounds: Identification of compounds present in the plant extract was performed using National Institute Standard and Technology (NIST) database. Interpretation of mass spectrum GC-MS was done using NIST library having more than 62000 patterns. Spectrum of unknown compounds was compared with the spectrum of known components stored in NIST library. Then the name, molecular weight and structure of the compounds of test sample were determined.

GC-MS spectrum (Fig.1) of methanol extract of Cuscuta reflexa grown on Acacia nilotica host plant confirmed the presence of different bio molecules along with chemical structure of bioactive compounds present in the crude plant extract (Table-1) that are responsible for the medicinal properties of sample plant.

\section{Conclusion}

The present study helps to predict the chemical formula and structure of different natural compounds present in the crude extract of Cuscuta reflexa. The existence of various bioactive compounds in the Cuscuta reveals its potent ability as being medicinal plant in the Ayurveda. Further investigation may lead to isolation, identification and structure elucidation of pure bioactive compounds from Cuscuta reflexa and screening of its pharmacological activity will be supportive for further drug development.

\section{REFERENCES}

1. J.H. Dawson, L.J. Musselman, P. Wolswinkel and I. Dorr, Rev. Weed Sci., 6, 265 (1994).

2. L.V. Costa-Lotufo, M.T.H. Khan, A. Ather, D.V. Wilke, P.C. Jimenez, C. Pessoa, M.E.A. de Moraes and M.O. de Moraes, J. Ethnopharmacol., 99, 21 (2005); https://doi.org/10.1016/j.jep.2005.01.041.

3. B.P. Ezhilan and R. Neelamegam, Pharmacogn. Res., 4, 11 (2012); https://doi.org/10.4103/0974-8490.91028.

4. L.K. Kanthal, A. Dey, K. Satyavathi and P. Bhojaraju, Pharmacogn. Res., 6, 58 (2014); https://doi.org/10.4103/0974-8490.122919.

5. N.R. Bhalodia and V.J. Shukla, J. Adv. Pharm. Technol. Res., 2, 104 (2011); https://doi.org/10.4103/2231-4040.82956. 